\title{
Mobile CARS - IRS Instrument for Simultaneous Spectroscopic Measurement of Multiple Properties in Gaseous Flows
}

\author{
D. Bivolaru', J. W. Lee ${ }^{2}$, S. B. Jones ${ }^{2}$, S. Tedder ${ }^{3}$, P. M. Danehy ${ }^{4}$ \\ NASA Langley Research Center, Hampton, VA 23681 \\ M. C. Weikl ${ }^{5}$, Universität Erlangen-Nürnberg, Erlangen, Germany
}

G. Magnotti ${ }^{6}$, and A. D. Cutler ${ }^{7}$, The George Washington University, Newport News, VA 23602

\begin{abstract}
This paper describes a measurement system based on the dual-pump coherent anti-Stokes Raman spectroscopy (CARS) and interferometric Rayleigh scattering (IRS) methods. The IRS measurement is performed simultaneously with the CARS measurement using a common green laser beam as a narrow-band light source. The mobile CARS-IRS instrument is designed for the use both in laboratories as well as in ground-based combustion test facilities. Furthermore, it is designed to be easily transported between laboratory and test facility. It performs single-point spatially and temporally resolved simultaneous measurements of temperature, species mole fraction of $\mathrm{N}_{2}, \mathrm{O}_{2}$, and $\mathrm{H}_{2}$, and two-components of velocity. A mobile laser system can be placed inside or outside the test facility, while a beam receiving and monitoring system is placed near the measurement location. Measurements in a laboratory small-scale Mach $1.6 \quad \mathrm{H}_{2}$-air combustion-heated supersonic jet were performed to test the capability of the system. Final setup and pretests of a larger scale reacting jet are ongoing at NASA Langley Research Center's Direct Connect Supersonic Combustor Test Facility (DCSCTF).
\end{abstract}

\section{INTRODUCTION}

Non-intrusive optical instrumentation systems capable of obtaining measurements of multiple fluid properties in a single laser pulse have been used to characterize high speed and combusting flows. For example, researchers have employed CARS to measure temperature [1], dual-pump CARS to measure temperature and species mole fraction [2], and Rayleigh scattering $[\underline{3}, \underline{4}]$ to measure temperature, density, and velocity in combusting flows. When multiple flow properties are measured simultaneously, correlations between those properties can be used to obtain a detailed understanding of complex flow behavior. These correlations can aid in the development of multi-parameter turbulence models for computational fluid dynamics codes. However, past work has generally been limited to independent measurements of temperature and species concentration using CARS and measurements of velocity using a variety of methods including interferometric Rayleigh scattering. We are developing a diagnostic system for spatially and temporally resolved simultaneous measurements of gas composition, temperature and multiple components of velocity in gas flows. In Reference [5], we reported the combination, for the first time in a laboratory, of a dual-pump CARS system [2] with an interferometric Rayleigh scattering (IRS) system [6] ], to provide time-resolved simultaneous measurement of temperature, species concentration of $\mathrm{N}_{2}, \mathrm{O}_{2}$, and $\mathrm{H}_{2}$, and two components of velocity in gaseous media. References [7] and [8] present single-point measurements performed with the CARS-IRS system to demonstrate its capability in a laboratory-scale Mach $1.6 \quad \mathrm{H}_{2}$-air combustion-heated supersonic reacting jet.

\footnotetext{
${ }^{1}$ NASA Postdoctoral Fellow, Advanced Sensing and Optical Measurement Branch, MS 493, Daniel.Bivolaru-1@nasa.gov

2 Electro-optical technician, Advanced Sensing and Optical Measurement Branch, MS 493

${ }^{3}$ Research Assistant, College of William and Mary, Williamsburg, VA 23669

${ }^{4}$ Senior Research Scientist, Advanced Sensing and Optical Measurement Branch, MS 493

${ }^{5}$ Research Assistant

${ }^{6}$ Research Assistant

${ }^{7}$ Professor

Disclaimer: The mention of specific products in this paper does not constitute an official endorsement of those products. The views and opinions expressed in this paper are the authors, and do not represent the official position of NASA or the above named Universities.
}

This work was supported by the NASA Fundamental Aeronautics Program - Hypersonics Project, Experimental Capabilities and Propulsion Disciplines and Mr. George Rumford, program manager of the Defense Test Resource Management Center (DTRMC) Test and Evaluation/Science and Technology (T\&E/S\&T) program, under the Hypersonic Test focus area. Markus C. Weikl would like to thank the Erlangen Graduate School in Advanced Optical Technologies (SAOT) for financial support. 
This paper describes a compact and versatile mobile version of this system designed primarily for the use in various largescale test facilities and laboratories at NASA Langley Research Center. These large-scale experiments are needed to generate spatially and temporally resolved experimental data for the CFD modelers. The final setup and pretests of a large-scale axisymmetric Mach $1.6 \mathrm{H}_{2}$-air reacting jet are ongoing at NASA Langley Research Center's Direct Connect Supersonic Combustor Test Facility (DCSCTF).

This system was designed based on lessons learned from other mobile laser diagnostics systems for large-scale facilities, as for example Refs. [9]], [10], and [11].

\section{THE CARS-IRS INSTRUMENT}

The CARS-IRS instrument consists of a mobile laser system (MLS) hosting the main and auxiliary lasers, and the laser delivery optics (Fig. 1), and a beam receiving system (BRS) that is placed near the measurement location. The BRS directs and moves the probing laser beams into the flow and collects and transmits the resulting CARS and IRS optical signals to their respective detectors (Fig. 2). The MLS has a fixed design (with flexibility for further development) and it can be placed inside or outside the test facility while the BRS is designed for the specific experiment to be performed. In fact, two BRS systems have been used: one in the laboratory and a second one in the large-scale facility.

\section{Dual-pump CARS system}

For the measurement of temperature and the absolute mole fractions of $\mathrm{N}_{2}, \mathrm{O}_{2}$, and $\mathrm{H}_{2}$ we use a dual-pump CARS method $[\underline{1}, \underline{2}]$. The system uses a spectrally narrow green laser $(0.005$ $\mathrm{cm}^{-1}, 20 \mathrm{~Hz}, 9$ nsec/pulse, seeded Nd:YAG@ 532 nm - Spectra Physics PRO-290) and a tunable narrow-band yellow dye laser

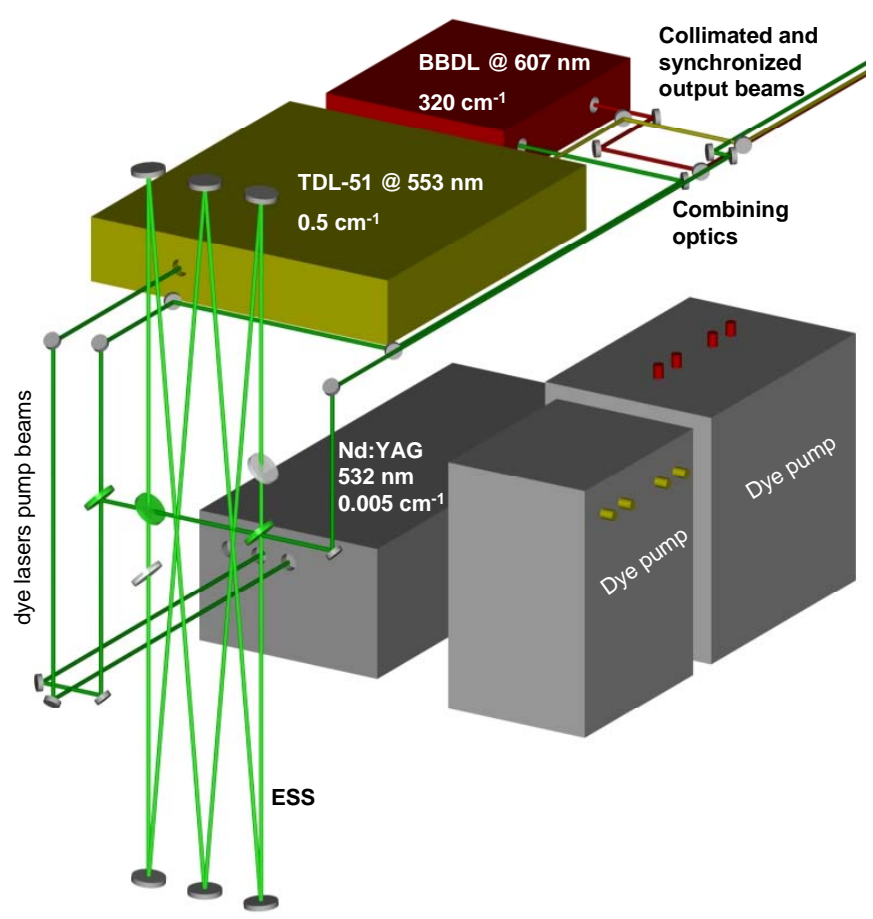

Figure 1. The optical setup of the mobile laser system (MLS) containing the main and auxiliary lasers and the beams delivery optics.

(Quantel TDL-51@ 553 nm, $0.5 \mathrm{~cm}^{-1}$ linewidth) as pump beams and a spectrally broad red tunable dye laser (in house designed, cavity-based oscillator BBDL @ 603 nm, $360 \mathrm{~cm}^{-1}$ ) beam as the Stokes beam. The optical arrangement of these lasers on the MLS is shown in Fig. 1 . The main green laser and the dye circulators are mounted on a lower level optical table while the red and yellow dye lasers are mounted on an upper level (the optical tables are not shown in the figure). Telescopes are used for yellow and red beams to increase the beam size and control the beam divergence. Delay lines are added to all beams to synchronize the laser pulses for the time of arrival at the output of the MLS.

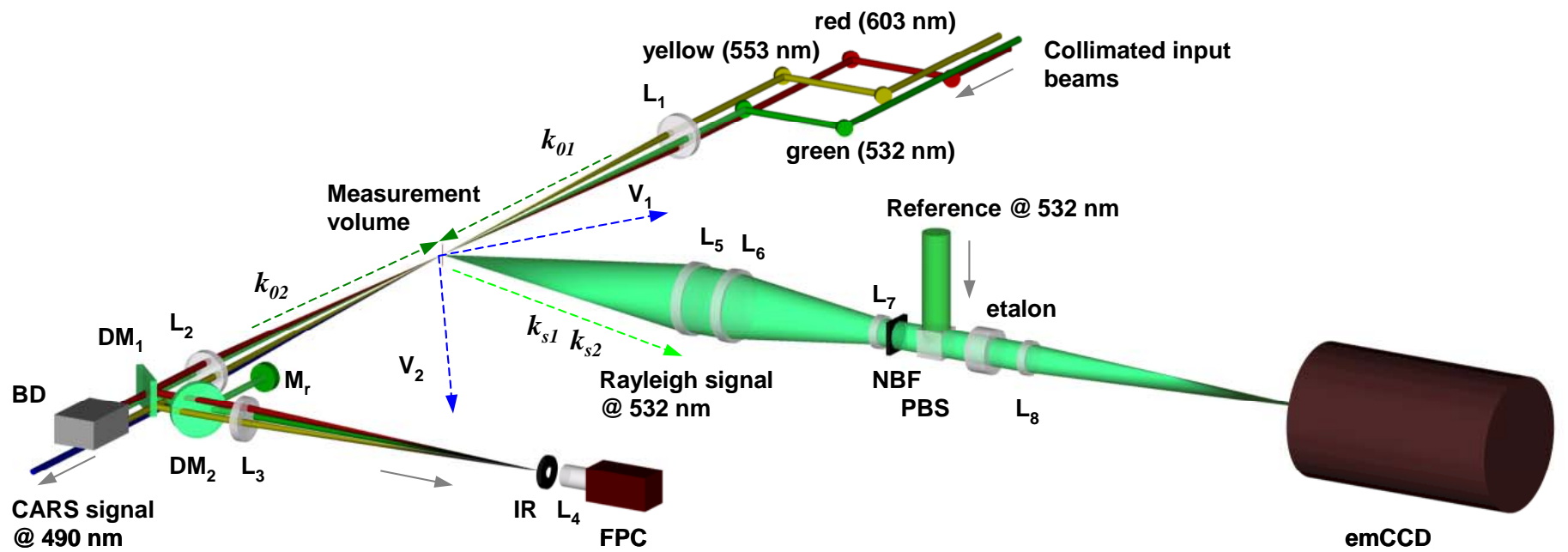

Figure 2. The transmitting and receiving optics of the CARS-IRS system 


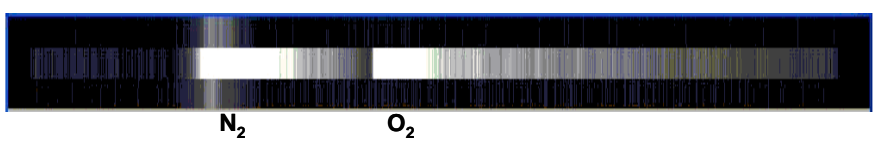

Figure 3. Single-shot image containing three horizontal bins, the CARS spectra (middle), and the background light (upper and lower bins), recorded in room air.

Referring to the optical setup of the BRS system shown partially in Fig. 2, the three laser beams of different colors from the output of the MLS are delivered by 5 broadband mirrors, $76.2 \mathrm{~mm}$ diameter mirrors (partially seen in Fig. 5(c)), with a total loss of the input beams energy of less than $45 \%$ in green, $17 \%$ in yellow, and $11 \%$ in red) and combined at the focusing point of lens $\mathrm{L}_{1}$ in a folded BOXCARS phase matching geometry to generate a blue spectrally-broad CARS signal beam. Spatial locations and parallelism of these beams at the input of the BRS (to obtain the phase matching geometry) are adjusted using the combining output optics of the MLS. To maximize the CARS signal and perform fine adjustments of beam crossing at the focus of lens $\mathrm{L}_{1}$ separate combining optics are used right before the lens. This assembly consists of three pairs of adjustable narrowband dichroic mirrors (used for higher efficiency), one pair for each color.

After passing through the measurement volume, the threecolor input beams plus the coherent CARS signal beam at 491 nm (blue) are collected and collimated by the lens $L_{2}$ after which the beam dump $\mathrm{BD}$ and dichroic mirror $\mathrm{DM}_{1}$ removes the pumps and Stokes beams. The mirror $\mathrm{DM}_{1}$ (with maximum reflectivity in green) reflects the green beam for further use in Rayleigh scattering. The CARS signal, which contains $\mathrm{N}_{2}, \mathrm{O}_{2}$ and $\mathrm{H}_{2}$ spectra, is passed through a narrow band interference filter to reject background light, and is focused by two "crossed" cylindrical lenses on the input slit of a one-meter spectrometer (McPherson) with a 2400 groove $/ \mathrm{mm}$ grating. At the output of the spectrometer, the CARS spectra are recorded by a Princeton Instruments PIXIS100B CCD camera with an area of 1340 pixels horizontally $x$ 100 pixels vertically. The shape of these spectra provides information on the temperature while the relative intensities of the different molecules spectra provide a measure of the relative mole fractions. Figure $\underline{3}$ shows a single-shot image in which signal is binned in the vertical direction to form three horizontal bins of 33 pixels each, the CARS spectra (middle), and background light (upper and lower bins), recorded in room air. During post-processing, the background bins are used to correct the CARS spectra for camera dark noise and unwanted background light.

Broadband Dye Laser: As mentioned, the dual-pump CARS system is configured to measure rotational vibrational $\mathrm{N}_{2}$ and $\mathrm{O}_{2}$ as well as pure rotational $\mathrm{H}_{2}$ lines in the same spectral region. To cover this spectral region, a broadband laser oscillator is required. We investigated a modified oscillator based on the design of Hahn et al [10], which can be operated in a "modeless" (MDO), i.e., no cavity, or a cavity (CDO) configuration.

Both oscillators, CDO and MDO, use a $4 \mathrm{~mm}$ diameter Bethune dye cell and a reflector, a parabolic mirror or a combination of a planar mirror and a lens to replace the mirror. The mirror collects a part of the stimulated emission coming from the cell and reflects it back to create a laser beam of amplified stimulated emission. Optical coupling with the next stage of amplification is performed using a pinhole for MDO or a $50 \%$ reflectivity mirror for $\mathrm{CDO}$. The length between the retro-reflector and optocoupler is $0.3 \mathrm{~m}$, and $1 \mathrm{~m}$ for the CDO and MDO, respectively. A glass plate mounted at the Brewster angle in front of the mirror is used to reject one polarization of the spontaneous emission. The laser medium for both lasers, having a length of $40 \mathrm{~mm}$, is a flowing solution of methanol and Rhodamine 640 laser dye. Both oscillators were sidepumped with a fraction of the narrow-band Nd:YAG laser at $532 \mathrm{~nm}$. The MDO configuration used a pump laser beam with energy of about $125 \mathrm{~mJ} /$ pulse. This beam was obtained from wavelength doubling of the infrared light usually dumped by the Nd:YAG laser. The output energy of this MDO oscillator was small (unmeasurable with our power meter), requiring multiple stages of amplification to obtain usable output energy. The CDO type oscillator was pumped with energy of about 30 $\mathrm{mJ} /$ pulse, generating about $3 \mathrm{~mJ} /$ pulse of laser radiation at its output. The pumping was performed by means of two parallel beams expanded by a telescope using cylindrical lenses. The beams were generated using the multiple reflection of a highenergy beam from both surfaces of an uncoated thick glass plate mounted at 45 degrees. This method produced two partially overlapped elliptical cross-section beams that permitted high efficiency pumping for this long dye cell. An amplifier stage was added to this cavity oscillator configuration consisting of a rectangular dye cell having parallel windows and collinear beam pumping optics. The flow of the dye solution (having a concentration of about $1 / 3^{\text {rd }}$ of that of the oscillator solution) is oriented at the Brewster angle to minimize losses. The amplifier is pumped with the fraction of the input pump beam that passes through the glass plate of the oscillator. The maximum output energy and wavelength are about $24 \mathrm{~mJ} /$ pulse and $607 \mathrm{~nm}$, respectively. Higher energy pumping of the amplifier is avoided due to intra-window lasing and dye saturation effects.

\section{Interferometric Rayleigh Scattering (IRS) System}

The interferometric Rayleigh scattering measurement system is similar to earlier versions that are described in References $[\underline{5}, \underline{6}$, and $\underline{7}]$. The same seeded green laser beam employed for CARS is used as a narrow-band light source for the Rayleigh system. The difference from the previous versions is that two components of velocity are measured directly and simultaneously. This is performed by redirecting the green laser beam back toward the measurement volume for a second 


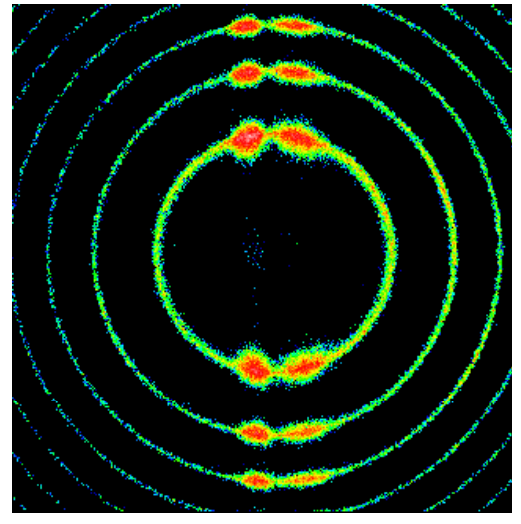

$\mathrm{V}_{2} \mathrm{~V}_{1}$

Figure 4. Single-shot interferogram obtained in a flame at $925 \mathrm{~K}$ containing Rayleigh scattering spectral information used to solve directly for two components of velocity.

measurement using the retroreflective mirror $\mathrm{M}_{\mathrm{r}}$ (Fig. 2). This reflected beam has a small offset from the optical axis so it can be dumped on the MLS before reaching the laser output port. The time delay between these two Rayleigh scattering measurements is set to more than 9 nsec (one FWHM) to minimize the influence of this beam on the ongoing CARS process. This is performed by adequate positioning of the dichroic mirrors $\mathrm{DM}_{1}$, and $\mathrm{DM}_{2}$, The light collected by the lenses $L_{5}$ in the direction of $\boldsymbol{k}_{s 1}$ (perpendicular to the laser beam and situated in the same plane as the laser beam and the flow axis) has a Doppler shift frequency given by $\Delta v_{1}=\left(\boldsymbol{k}_{s 1}-\right.$ $\left.\boldsymbol{k}_{01}\right) \cdot \boldsymbol{V}$, where $\boldsymbol{k}_{01}$ is the input laser beam wave vector, and $\boldsymbol{V}$ is the velocity vector. The direction of the vector $\left(\boldsymbol{k}_{s 1}-\boldsymbol{k}_{01}\right)$ is the direction of the velocity component, defined as $V_{1}$. The orientation of this vector relative to the flow exit plane is chosen to be in the radial direction of velocity, $V_{r}=\mathrm{V}_{1}$ of the flow. To measure the second component of velocity $V_{z}$ in the axial direction of the flow issuing from the burner, scattered light is collected by lens $L_{5}$ from the retroreflected probing beam in the direction of the wave vector $\boldsymbol{k}_{\mathrm{s} 2}$, where $\boldsymbol{k}_{02}=-\boldsymbol{k}_{01}$ is the retroreflected laser beam wave vector. The direction of $\left(\boldsymbol{k}_{\mathrm{s} 2}-\boldsymbol{k}_{02}\right)$ vector gives the direction of the velocity vector being measured, defined as $V_{2}$. Note that $V_{1}$ is perpendicular to $\mathrm{V}_{2}$. A similar arrangement was previously used by Eggins and Jakson [12] though his measurement system was time averaged. The light collected and collimated by the lenses $L_{5}$ is reduced in diameter and recollimated by the lenses $\mathrm{L}_{6}$, and $\mathrm{L}_{7}$, filtered by a narrow band filter (NBF) to remove the scattered light from the yellow and the red CARS beams, combined with a fraction of the laser light using the beam combiner (PBS), and passed through a solid etalon. An electron multiplication (em) gain CCD camera (Princeton Instruments Photon Max 512 with an area of $512 \times 512$ pixels with $18 \mu \mathrm{m}$ square pixels) records the interference fringe pattern, which is formed at the output of the etalon by the lens $\mathrm{L}_{8}$. This pattern contains the scattered light information from multiple collecting angles, the reference laser frequency (concentric ring pattern) as well as the laser energy information (the amplitude of the concentric ring pattern). However, if the mirror $\mathrm{M}_{\mathrm{r}}$ is oriented perpendicular to the optical axis, the spectra of scattered light from both collection directions overlap on the interferogram. Then, the interference pattern corresponding to two different velocity components cannot be separated. This ambiguity can be removed if the experiment is designed so that one measured velocity component is always much bigger than the other (for example, the axial velocity component in a high-speed jet is generally much larger than the radial component). An alternative way to remove this ambiguity is to slightly change the angle of the retro reflector $\mathrm{M}_{\mathrm{r}}$. The new image contains two parallel patterns situated symmetrically around the center of the interferogram, as shown in the example interferogram of Fig. 4. Each of the vertical fringe patterns in the image plane represents a measurement direction, e.g. a velocity component. The concentric ring pattern is the interferogram produced by the reference laser. For the spectrum shown the gas temperature is about $925 \mathrm{~K}$. Image processing software extracts the molecular scattering spectral information from interferograms (see references [] for details).

The Rayleigh interferograms and the CARS spectra are acquired simultaneously in two separate files on separate computers for each laser pulse by synchronizing the cameras with the green laser Q-switch at $20 \mathrm{~Hz}$. Post processing of data is performed to fit CARS and Rayleigh spectra with theoretical models, thereby determining the gas temperature and the species mole fractions of $\mathrm{H}_{2}, \mathrm{O}_{2}$, and $\mathrm{N}_{2}$, and the Doppler shift frequency of the Rayleigh signal which is used to solve for two components of velocity (for details of these analyses see Refs [1] and [ㅁ]).

Energy Storage System: The Rayleigh signal intensity is linearly proportional to the laser energy but the breakdown of the gas at room temperature limits the use of the laser energy for CARS or Rayleigh scattering to about $65 \mathrm{~mJ} / \mathrm{pulse}$ when using $410 \mathrm{~mm}$ focal length lenses to focus the light. To increase the Rayleigh signal and to avoid laser-induced breakdown and saturation effects in CARS, a fraction of the green laser energy is delayed $18 \mathrm{nsec}$ and re-injected on the same optical axis by using an energy storage system (ESS) which contains a pulse stretcher. The optical arrangement shown in Fig. 1 , and described in Reference [ [ $]$ ], is designed to obtain a burst of three high-energy laser pulses of almost identical amplitudes and secondary pulses of low energy. Basically, two beam splitters with a transmittance of $68 \%$ and $38 \%$ are used as input and output combiners respectively, and six flat mirrors create three delay lines of $0.2 \mathrm{~m}$ (between beam splitters), $2.9 \mathrm{~m}$ (from the first to the second beam splitters), and $2.9 \mathrm{~m}$ (from the second to the first beam splitters) respectively. Two glass windows are used in conjunction with the beam splitters to compensate for the changes in optical path by refraction. At full input laser energy of about $300 \mathrm{~mJ} /$ pulse, the storage system can deliver at its output up to $200 \mathrm{~mJ} / \mathrm{pulse}$. 


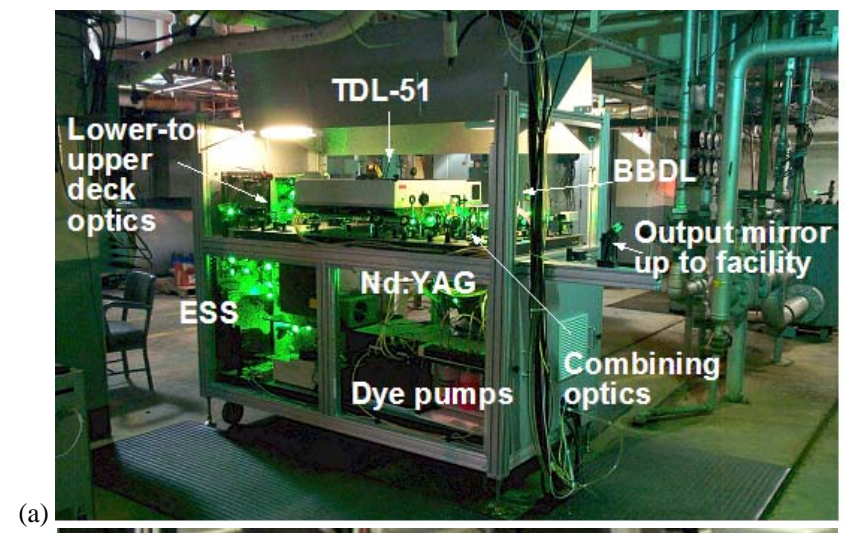

(a)

(b)

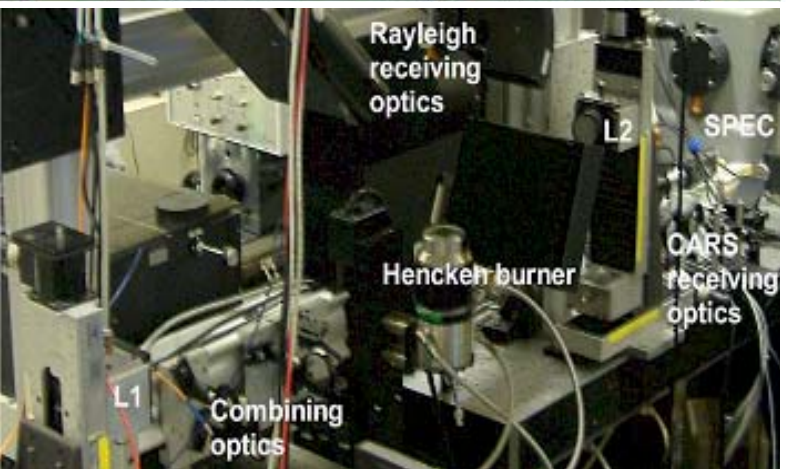

(c)

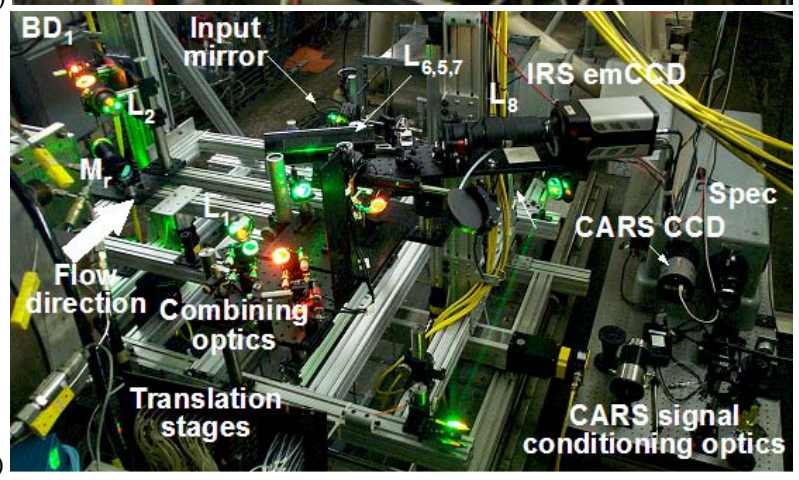

Figure 5. Image of the CARS-IRS instrument arrangement: (a), the mobile laser system (MLS) housing lasers and laser delivery optics, and (b), (c) the beam receiving systems (BRS) for the laboratory and the test facility respectively.

\section{Laser Beam Viewing System}

To monitor the CARS and Rayleigh laser beams in real time and to perform the laser beams monitoring on the cart and the optimization of beams crossing at the measurement volume we used focal plane imaging onto a video camera [13]. This monitoring system is denoted here as the CARS and Rayleigh beam viewing (CRB-View) system. For the CRB-View system arrangement shown in Fig. $\underline{2}$ a real image of the beams at the sample region is produced on the video camera (FPC). This particular setup does not waste input beam energy to generate this image.

To analyze the beam crossing, overlap, and profiles, the output beams, which are usually ignored in a typical CARS -
IRS application, are isolated from the outgoing CARS signal beam using the rectangular dichroic mirror $\mathrm{DM}_{1}$ (maximum reflectivity in green) and refocused by lens $\mathrm{L}_{3}$ (identical focal length as $L_{1}$ and $L_{2}=410 \mathrm{~mm}$ ). The mirror $\mathrm{DM}_{1}$ is placed above the signal beam path to avoid loss in signal. A second dichroic mirror $\mathrm{DM}_{2}$, centered at $532 \mathrm{~nm}$ with maximum reflectivity at $45 \mathrm{deg}$, further reduces the green beam energy. Angular tuning of this mirror around the maximum reflectivity position allows adjusting the transmitted energy toward the FPC camera. The green beam reflected by the mirror $\mathrm{DM}_{2}$ is directed toward the retroreflector $\mathrm{M}_{\mathrm{r}}$ for IRS use. Depending on the beam energy, neutral density filters (not shown) can be introduced in the optical path to equalize the beams' intensity before reaching the FPC camera. A microscope objective lens $\mathrm{L}_{4}$ (48 mm, chosen for the desired magnification) is placed near the focal region of lens $\mathrm{L}_{3}$ to form a magnified image of the crossing beams region on the FPC camera. The iris, IR, is used to remove stray light and secondary images of the beams that are formed by the dichroic mirror $\mathrm{DM}_{1}$. Different planes near the crossing point of the beams can be observed as the lens $\mathrm{L}_{4}$ and the camera are simultaneously translated parallel to the optical axis.

Similarly, the leakage of the laser beams through one of the broadband mirrors used to direct the beams from MLS to BRS is used as input for a secondary CRB-View system. This system performs the monitoring of the parallel laser beams at the input of the BRS system (before the measurement location, focusing lens $\mathrm{L}_{1}$ ).

Monitoring of the beams intensity profiles and crossing permitted the beams to be adjusted in real time during both preliminary setups and experiments.

\section{Hardware Configuration}

The mobile CARS-IRS instrument has been used with two different optical configurations of the BRS: one for laboratory use and another for the DCSCTF. For laboratory use, the flow under investigation is oriented vertically and the combustion experiment hardware itself can be translated in three dimensions. In the DCSCTF, the facility nozzle is fixed horizontally, so the BRS translates in three dimensions to probe multiple spatial locations. Fig. 5(a) shows the image of the MLS, while the laboratory and facility BRS's are shown in the images of Fig. 5(므) and Fig. 5(ㅁ), respectively.

The facility BRS surrounds the test flow to be investigated and provides many mounting options to maximize flexibility as needed for reconfiguring the optical system as a function of environmental constrains. The BRS has a base that measures approximately $1.5 \mathrm{~m}^{2}$ and stands approximately $2 \mathrm{~m}$ tall. Three linear translation stages (Parker Motor Control Systems) powered by servomotors provide the means of translating the CARS-IRS measurement location up to $0.6 \mathrm{~m} \times 0.6 \mathrm{~m}$ area in the vertical and cross flow directions, and $1 \mathrm{~m}$ in the upstreamdownstream direction function of the experiment 


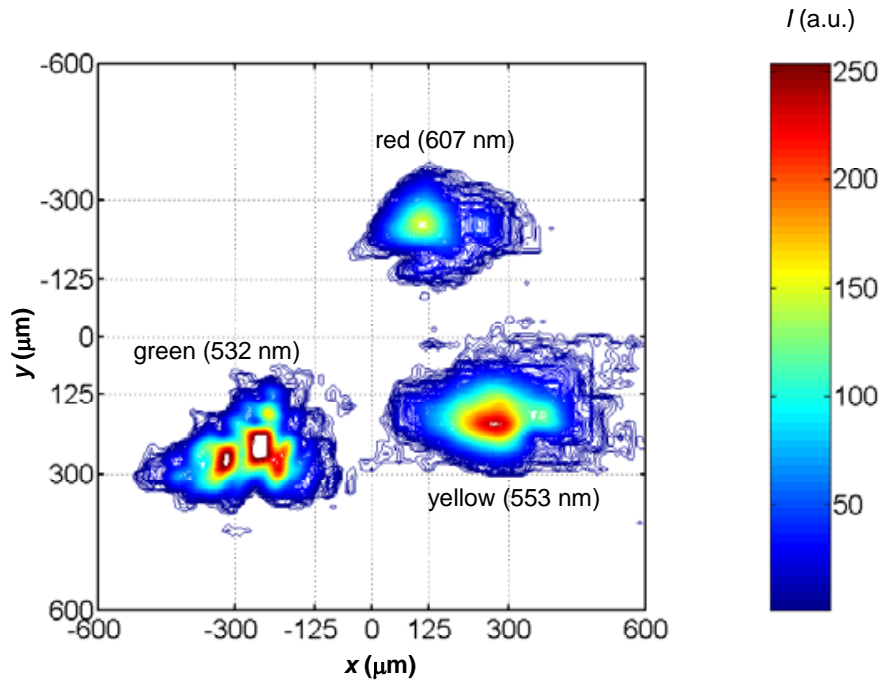

Figure 6. Laser beam profiles of simultaneous, single-point, dual-pump CARS and IRS: The spatial location of the three input beams is in a plane transverse to the optical axis located about $8 \mathrm{~mm}$ from the center of the measurement volume. The $532 \mathrm{~nm}$ pump beam consists of multiple collinear pulses originating from the pulse stretcher (ESS).

configuration. The current stages have a position accuracy of $25 \mu \mathrm{m}$ and repeatability of $\pm 3 \mu \mathrm{m}$ in the vertical direction and $200 \mu \mathrm{m}$ and $\pm 12 \mu \mathrm{m}$ respectively in the cross flow and upstream-downstream directions.

The user interface to the translation stage is through a personal computer connected to a motor controller and amplifier subsystem. The program control for the BCS gives the user two options for positioning the system. The first is a single coordinate input $(\mathrm{x}, \mathrm{y}, \mathrm{z})$ motion that commands the translation stages to move at constant, preprogrammed velocity, to an absolute position to perform a measurement. The second type uses a text input file of multiple positions plus a dwell time at each location, to generate a program that is sent to and executed by the motor controller. When executed, the position data generated by the motor encoders can be synchronized with an external event such as the laser Q-switch trigger pulse. An output file is then generated with the position data written along side of the trigger index. This provides the means to correlate the CARS-IRS data to the specific coordinates measured in the flow.

The MLS is constructed primarily of $76.2 \times 76.2 \mathrm{~mm}$ Tslotted extruded aluminum framing. The cart measures approximately $1.8 \mathrm{~m}$ high by $1 \mathrm{~m}$ wide and $2.2 \mathrm{~m}$ long. It contains two horizontally mounted optical tables that carry the lasers and associated optical hardware and a third that is vertically mounted to hold lower-to-upper table periscopes and the ESS. Six removable panels and four large hinged panels allow easy access to all of the internal components from any side. The entire cart is transportable on wheels or stabilized on adjustable feet. The MLS frame including three optical tables weigh approximately $680 \mathrm{~kg}$ and about $900 \mathrm{~kg}$ total with all of the lasers and hardware installed. The design of MLS frame is mainly based on a successful portable laser-induced fluorescence (PLIF) imaging system that has been developed for use in NASA Langley's wind tunnels for hypersonic flow visualization [9].

The CARS-IRS instrument is controlled by electronics mounted on a movable rack system containing: the personal computers to acquire and store measurement data; the main display, keyboard, and electronics modules for remote monitoring; instruments to control and measure the lasers wavelength, the timing of the laser Q-switch and lamps, and to synchronize the camera acquisitions for CARS and Rayleigh with the laser pulse; and the motor controller and amplifier subsystem for the linear translation stages. Software applications on each computer are run simultaneously or independently depending on the specific task.

\section{MeAsurements}

1) Laser beams profiles: Measurements of laser beam profiles using the CRB viewing system are shown in Fig. $\underline{6}$. The transverse plane image shows the three input CARS beams and the secondary green beams (used for the Rayleigh scattering signal enhancement to measure one component of velocity) as they pass through the measurement region. The contour lines represent curves of constant intensity using an arbitrary intensity scale. The white color in the center of the peak indicates CCD saturation. The focal length of $L_{1}$ is 410 $\mathrm{mm}$, the collimated beam diameters are about $10 \mathrm{~mm}$ (at maximum aperture) on the lens $\mathrm{L}_{1}$, and the focused beam diameters vary approximately from 50 to $100 \mu \mathrm{m}$, depending on wavelength. These measured beam diameters were based on a calibration (6 $\mu \mathrm{m} / \mathrm{pixel})$ using a slit of known width positioned at the focus point of lens $L_{1}$ (measurement location).

For illustration purposes the green, yellow and red beams are imaged out of focus in a plane about $8 \mathrm{~mm}$ from the center of the measurement volume. The beams of different color are separated by about $500 \mu \mathrm{m}$ at this location. The green beams form a cluster in the bottom left hand of the image; the beam which occurs first in time is of highest intensity and is used for both CARS and Rayleigh: the remaining grean beams are coming from the ESS and are used only for the Rayleigh system. This device reveals if the beams from the pulse stretcher are collinear or out of alignment. This avoids collecting Rayleigh scattered photons from different spatial locations, which would result in an undesirable spatial averaging of the measurement and/or in loss of signal. The image shows the spatial non-uniformity produced by overlapping the secondary laser beams coming from the ESS.

In addition to beam crossing alignments for CARS and beam overlapping for IRS, the CRB viewing system has the capability of monitoring laser beam steering. For example, we readily observed small fluctuations in beam intensities and spatial locations during supersonic flow experiments due to environmental vibrations and gas refractive index fluctuations. 


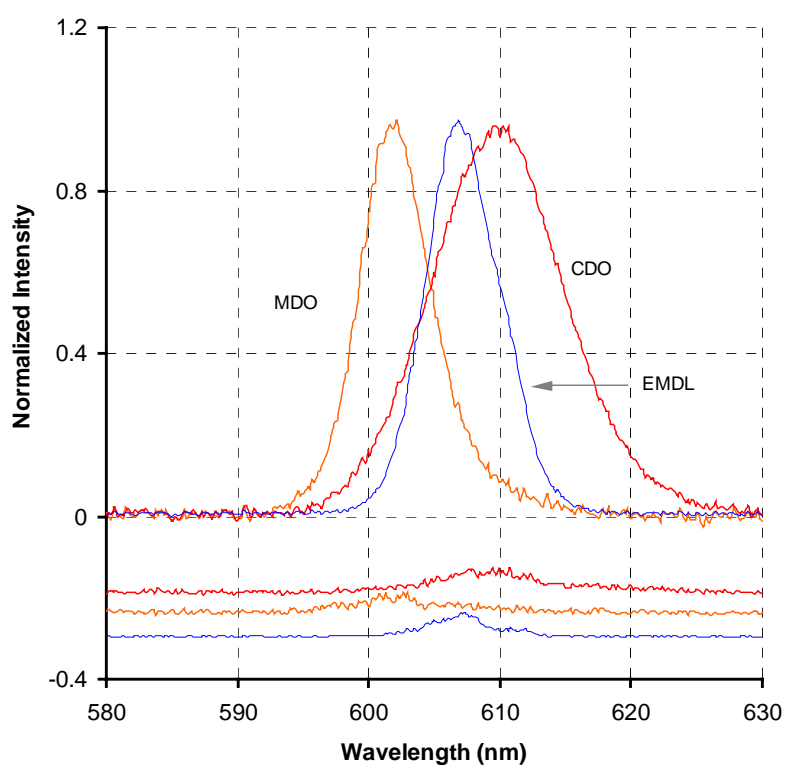

Figure 7. Spectral comparison of broadband dye lasers.

The variation of beam intensity along the optical axis can be obtained by performing multiple transverse profiles at incremental locations and then post processing. The maximum peak intensity values for each beam obtained from these profiles determine these functions.

\section{2) Stokes Laser Spectral Characteristics:}

Measurements of spectral characteristics of the Stokes laser are shown in Fig. $\underline{7}$. A low-resolution spectrometer (600 lines $/ \mathrm{mm}$ grating blazed at $300 \mathrm{~nm}$ - Ocean Optics Model USB4000-UV-VIS/OFLV4-200-850 with $25 \mu \mathrm{m}$ slit) was used for this measurement, with the CDO and MDO laser optical setup having the same dye cell, dye solution, retro-reflector, and pumping energy. The variation from its mean for each case, obtained from ten consecutive measurements, is shown with a constant bias in the lower part of the figure. In comparison with a commercially available Ewart's modeless dye laser (EMDL) [14] (blue trace) tested in our laboratory, the cavity configuration shows a broader spectral profile, while the MDO has about the same FWHM. The FWHM is $6 \mathrm{~nm}$ for the MDO and 10\% higher for EMDL and about $12 \mathrm{~nm}\left(320 \mathrm{~cm}^{-1}\right)$ for the CDO oscillator, i.e. the bandwidth of the spectral profile for the cavity laser is about $50 \%$ larger than that of a modeless dye laser. These spectra widths increase by about $15 \%$ when a mixture of Rhodamine 610 and 640 is used as the dye solution. A wavelength shift of the spectral profile of about $8 \mathrm{~nm}$ is also obtained when switching between these two laser output coupler configurations. Hence, comparison of CARS spectra by simply replacing the optocoupler with a pinhole is not possible without modifying the dye concentration for one configuration to account for the wavelength shift of the other.

The spectra display a maximum standard deviation from its mean of about $5.7 \%$ for MDO and an increased value of $6.7 \%$ for CDO. These fluctuations are higher than those reported in literature for $\mathrm{N}_{2}$ CARS work using MDO's [10, and 14]. The advantage of using broader Stokes spectra is to increase the CARS signal-to-noise level at the edges of spectra (where the rotational hydrogen lines $S(5)$ and $S(6)$ are located), increasing the precision of temperature measurement and the accuracy of hydrogen mole fractions measured. The biggest disadvantage of the CDO is the cavity mode competition that leads to a worse precision of CARS temperature measurements if compared with systems using MDO's ([10, and 14]). Another disadvantage of using such a cavity dye oscillator (CDO) design is that it requires a longer delay line ( $\sim 3$ nsec) to synchronize the oscillator output and the dye amplifier pump beams. Consequently the other two CARS pump beams require longer delay lines for temporal matching, complicating the system, decreasing its efficiency, and increasing its sensitivity to vibrations.

3) CARS-IRS Measurements: Although tests of the newer version of the system are in progress and preliminary data are available, measurements examples performed with the laboratory setup in a small-scale Mach $1.6 \mathrm{H}_{2}$-air combustionheated supersonic jet are presented herein $[\underline{7}, \underline{8}]$. Each spectra of CARS and IRS in Fig. $\underline{8}$, and Fig. $\underline{9}$, respectively shows the experimental data, the theoretical fit, and the residual between them.

The measurement volume is a single point for CARS of about $1.5 \mathrm{~mm} \times 0.1 \mathrm{~mm} \times 0.1 \mathrm{~mm}$ and up to four non-evenly spaced points for Rayleigh scattering of about $0.1 \mathrm{~mm}^{3}$ each. These non-evenly spaced points are distributed across about $1.5 \mathrm{~mm}$. The beam energies at the measurement volume for these measurements were in the range of 150 to $200 \mathrm{~mJ} / \mathrm{pulse}$ for the green laser burst, with $65 \mathrm{~mJ} /$ pulse in the first green pulse used for CARS, 2 to $10 \mathrm{~mJ} /$ pulse for the red, and 5 to $15 \mathrm{~mJ} /$ pulse for the yellow laser beam.

Figure $\underline{8}$ shows an example of dual-pump CARS spectra measured on the axis of the jet at $70 \mathrm{~mm}$ (7 nozzle diameters) downstream of the nozzle exit. For this measurement we used the commercial EMDL [14] (with an additional stage of amplification) as the Stokes beam having a FWHM of about $220 \mathrm{~cm}^{-1}$. The increase in the noise at the edges of the CARS spectra are caused by low energy probing at the edges of the broadband spectrum where the $\mathrm{H}_{2}$ lines $\mathrm{S}(5)$ and $\mathrm{S}(6)$ are located. The relative measurement inaccuracy for the concentration of $\mathrm{H}_{2}$ in this work can be overcome by higher energy output and broader Stokes spectra of the CDO design (with a bandwidth of 320 to $360 \mathrm{~cm}^{-1}$ ).

The velocity measurements were performed simultaneously with the CARS measurements (examples are given in Fig. 4 of Ref. []]). However, both systems can be run independently if desired. Spatially resolved single-pulse spectra of interferometric Rayleigh scattering are shown in Fig. $\underline{9}$. These two spectra, extracted from interferograms similar to the interferogram presented in Fig. 4 show the Doppler shift of the Rayleigh signal with respect to the reference laser frequency, indicated by narrow peaks in Fig. 9(a) and black crosses in Fig. 9(b). These shifts are used to calculate two components of 


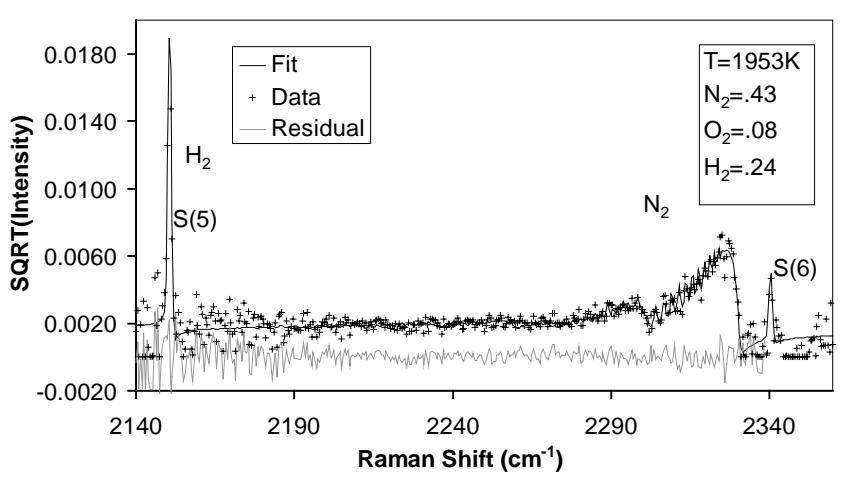

Figure 8. Single laser pulse spectra of CARS in a small-scale Mach $1.6 \mathrm{H}_{2}$-air combustion-heated supersonic jet used to determine the gas temperature and the species mole fractions of $\mathrm{H}_{2}, \mathrm{O}_{2}$, and $\mathrm{N}_{2}$.

velocity at two spatial locations about $0.4 \mathrm{~mm}$ apart. All fit functions are Gaussian with the narrow peak at the laser frequency. The standard deviation for the directly measured velocity component is smaller than $27 \mathrm{~m} / \mathrm{sec}$ at the highest measured temperature $(2380 \mathrm{~K})$ with a minimum of $8 \mathrm{~m} / \mathrm{sec}$ in stagnant air $(293 \mathrm{~K})$. The standard deviation is about $1 \%$ of the dynamic range of the IRS instrument. When the measurement of the velocity component is performed indirectly (that is, if two non-orthogonal velocity components are measured simultaneously), the standard deviation of the calculated component increases due to error propagation. A number of 400 consecutive interferograms containing the spectral information from two viewing directions and reference wavelength (green laser) were used for these calculations.

The single shot interferograms were obtained with the IRS system located close to the experiment (less than $2 \mathrm{~m}$ away) in a harsh environment, having a very high level of noise $(\sim 140$ $\mathrm{dB}$ ) and induced vibrations.

\section{CONCLUSIONS}

We described a mobile measurement system based on the coherent anti-Stokes Raman spectroscopy (CARS) and interferometric Rayleigh scattering (IRS) methods. The mobile CARS-IRS instrument was useful for studying combustion in laboratories and is in the process of testing in large-scale ground-based combustion test facilities. It performs singlepoint simultaneous measurements of temperature, species mole fraction of $\mathrm{N}_{2}, \mathrm{O}_{2}$, and $\mathrm{H}_{2}$, and two-components of velocity. The measurement system can be placed inside or outside the test facility while a beam-receiving system is placed near the measurement location. The measurement volume can be scanned spatially to generate a three-dimensional map of the flow.

For the measurement of temperature and the absolute mole fractions we used a dual-pump CARS method. A cavity dye oscillator was chosen for the Stokes laser rather than a "modeless" laser without cavity because of its higher energy

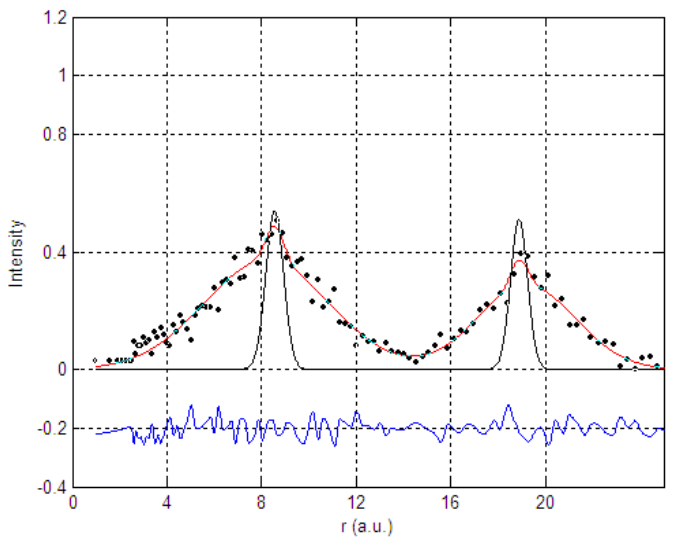

(a)

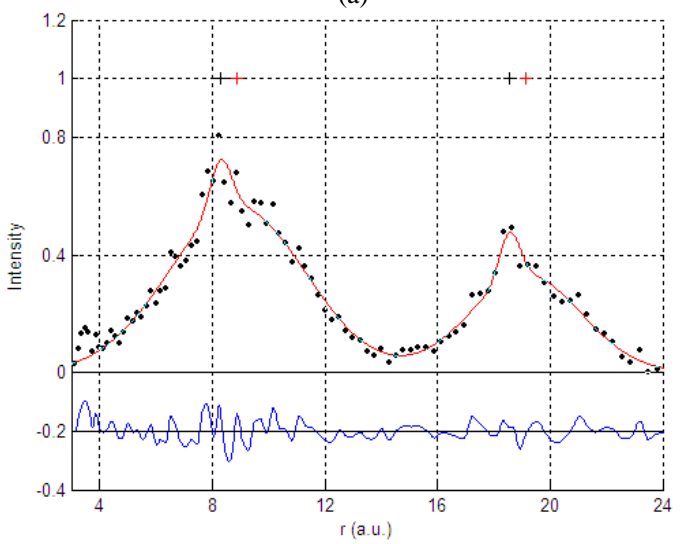

(b)

Figure 9. Simultaneous single laser pulse spectra of interferometric Rayleigh scattering obtained in a small-scale Mach $1.6 \mathrm{H}_{2}$-air combustionheated supersonic jet. These spectra are used to determine two-components of velocity.

output and spectral linewidth. In comparison with a modeless dye laser of similar design [10] the cavity laser also shows a broader spectral profile. The FWHM of the spectral profile for the cavity laser of $12 \mathrm{~nm}\left(320 \mathrm{~cm}^{-1}\right)$ is about $50 \%$ broader than that of a modeless dye laser having the same dye cell, dye solution, retro-reflector, and pumping energy. This width increases by about $15 \%$ when a mixture of Rhodamine 610 and 640 is used as the dye solution. The spectra fluctuate slightly with a maximum standard deviation of about $6.7 \%$.

Simultaneously with CARS measurements, an interferometric Rayleigh scattering system is used for the direct measurement of two components of velocity. Interferograms were obtained, containing the scattered light information from multiple collecting angles, the reference laser frequency as well as the laser energy information. The standard deviation for the directly measured velocity component is smaller than 27 $\mathrm{m} / \mathrm{sec}$ at the highest measured temperature $(2380 \mathrm{~K})$ with a minimum of $8 \mathrm{~m} / \mathrm{sec}$ in stagnant air $(290 \mathrm{~K})$.

We used focal plane imaging (CRB-View) to monitor the intensity profiles of the IRS laser beams in real time and to perform optimization of the CARS beams crossing. The system imaged the laser beams in the vicinity of the measurement volume. Beam parallelism, intensity profiles, crossings, and 
overlaps were monitored, allowing beam adjustments in real time during preliminary setup and experiments.

Measurements in a small-scale Mach $1.6 \mathrm{H}_{2}$-air combustionheated supersonic jet were performed in the laboratory to test the capability of the system. The installation in a large-scale test facility is ongoing.

\section{ACKNOWLEDGMENTS}

We thank Lloyd G. Wilson for installing and operating the laboratory-scale combustion flow hardware and James Downey for the development of the laser beams positioning system software. We also thank Diego Capriotti and Barry Lawhorne and his team for their technical support and preliminary tests in DCSCTF at NASA LaRC.

\section{REFEENCES}

[1] A. D. Cutler, P. M. Danehy, R. R. Springer, S. O'Byrne, D. P. Capriotti, R. Deloach, "Coherent Anti-Stokes Raman Spectroscopic Thermometry in a Supersonic Combustor", AIAA Journal, Vol. 41, Num. 12, Dec. p. 2451-2459, (2003).

[2] S. O’Byrne, P. M. Danehy, S. A. Tedder, and A. D. Cutler, Dual-Pump Coherent Anti-Stokes Raman Scattering Measurements in a Supersonic Combustor AIAA Journal Vol. 45, No. 4, p. 922-933, April (2007).

[3] Seasholtz, R. G., Zupanc, F. J. and Schneider, S. J., "Spectrally Resolved Rayleigh Scattering Diagnostics for Hydrogen-Oxygen Rocket Plume Studies,” J. Propulsion and Power, Vol. 8, No. 5, 1992, pp. 935-942.

[4] Seasholtz, R. G., Buggele, A. E. and Reeder, M., "Instantaneous Measurements in a Supersonic Wind tunnel Using Spectrally Resolved Rayleigh Scattering," Proceedings of the International Symposium on Optical Science, Engineering and Instrumentation, Society of PhotoOptical Instrumentation Engineers, Bellingham, WA, 1995.

[5] Bivolaru, D., Danehy, P.M., Grinstead, K. D., Tedder S., Cutler A. D., "Simultaneous CARS and Interferometric Rayleigh Scattering," AIAA Aerodynamic Measurement Technology and Ground Testing Conference, AIAA-2006-2968, San Francisco, June 2006.

[6] Bivolaru, D., Ötügen, M. V., Tzes, A. and Papadopoulos, G., "Image Processing for Interferometric Mie and Rayleigh Scattering Velocity Measurements, AIAA Journal, Vol. 37, No. 6, pp. 688-694, 1999.

[7] Tedder, S., Bivolaru, D., Danehy, P.M., Weikl, M., Beyrau, F., Seeger, T., Cutler, A.D., "Characterization of a Combined CARS and Interferometric Rayleigh Scattering System and Demonstration in a Mach 1.6 Combustion-Heated Jet," AIAA-2007-0871, 45 ${ }^{\text {th }}$ Aerospace Sciences Meeting and Exhibit, Reno, NV, 2007.

[8] A. D. Cutler, G. Magnotti, R. Baurle, D. Bivolaru, S.Tedder, and P. M. Danehy, "Development of Supersonic Combustion Experiments for CFD Modeling," AIAA Paper 2007-0000, 45th AIAA Aerospace Sciences Meeting and Exhibit, Reno, Nevada, Jan. 8-11, 2007.

[9] J. A. Wilkes, D.W. Alderfer, S. B. Jones, and P.M. Danehy, "Portable Fluorescence Imaging System for Hypersonic Flow Facilities," JANNAF Conference, Colorado Springs, Colorado, December 1-5, 2003,

[10] J. W. Hahn, C. W. Park, and S. N. Park, "Broadband coherent anti-Stokes Raman spectroscopy with a modeless dye laser," Applied Optics, Vol. 36, No. 27, pp. 6722-6728, 1997.

[11] T. J. Anderson, G. M. Dobbs, A. C. Eckbreth, "Mobile CARS instrument for combustion and plasma diagnostics," Applied Optics, Vol. 25, No. 22, pp. 4076-4085, 1986.

[12] P. L. Eggins and D. A. Jakson, "A two-component laser-Doppler anemometer using a single Fabry-Perot interferometer," Journal of Physics D: Applied Physics 8, No. 4 (11 March 1975) L45-L47.

[13] D. Bivolaru, G. C. Herring, "Focal-plane imaging of crossed beams in nonlinear optics experiments," Review of Scientific Instruments, 78, 056102 (2007).

[14] P. Ewart, “A Modeless, Variable Bandwidth, Tunable Laser," Optics Communications, Vol 55, pp. 124-126, 1985.

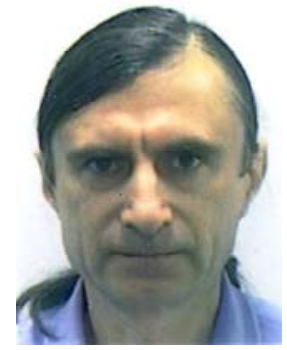

Daniel Bivolaru received his MS degree in Bucharest, Romania, in 1983. After graduation, he worked as an Engineering Physicist and a Research Scientist at the Institute for Nuclear Power Reactors, Pitesti-Colibasi, Romania, and as a Research Scientist at the Institute for Gravity and Space Sciences, Bucharest-Magurele, Romania. In 1996 he continued graduate studies in Aerospace Engineering as a Research Assistant in the Department of Mechanical, Aerospace and Manufacturing Engineering, Polytechnic University, USA. After receiving his Ph.D. in Mechanical Engineering in 2001, he continued his research at Polytechnic University as a Research Scientist.

His areas of interest are high-speed flows, plasma aerodynamics, and supersonic/hypersonic flow and plasma diagnostics. Since 2004 he is a NASA Postdoctoral Fellow at NASA Langley Research Center, Advanced Sensing and Optical Measurement Branch, Hampton, Virginia. Here his research is focused on laser diagnostics of gaseous flows using multi-point multi-property interferometric Rayleigh scattering; currently he develops the combined interferometric Rayleigh scattering - coherent anti-Stokes Raman spectroscopy (CARS) system to simultaneously measure temperature, species concentration and velocity in supersonic combustion flows.

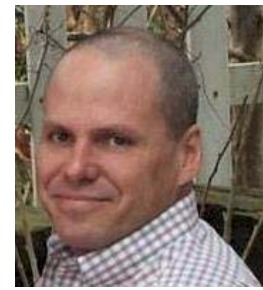

Joseph W. Lee received his Associate in Applied Science degree in Electronics Technology from Thomas Nelson Community College in 1981 and graduated the NASA Apprentice School in 1984. During his apprenticeship he assisted in the development and application of Laser Doppler Velocimetry (LDV) systems in various wind tunnels at NASA Langley Research Center (LaRC). After the NASA apprentice school he continued working in the area of LDV developing electronic systems and subsystems for signal conditioning, data acquisition and data simulation. In 1989 he became the lead technician for the research and development of Doppler Global Velocimetry (DGV). Joe was instrumental in advancing DGV from a laboratory curiosity to a flow measurement system capable of demonstrated velocity measurements. He has designed, installed and tested systems in facilities from small laboratory wind tunnels at LaRC to the large 40-by 80-foot National Full-scale Aerodynamic Complex at NASA Ames.

As an accomplished research technician he continues working in the areas of molecular filter based diagnostics, Rayleigh scattering, and CARS.

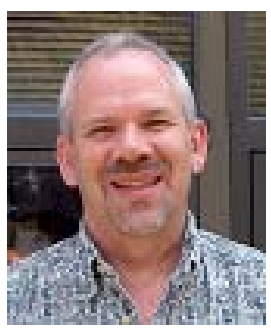

Stephen B. Jones is a Senior Electronics Technician assigned to the Advanced Sensing and Optical Measurements Branch of the Research and Technology Directorate at NASA Langley Research Center. Mr. Jones graduated from the NASA Apprentice program in 1982 and has been working at Langley for the past 30 years. In time, the majority of his work has been to develop flow visualization systems including Schlieren, shadowgraph and laser light sheet systems for the many different test facilities at NASA Langley. He has authored/coauthored more than 15 technical papers and 12 NASA Tech Briefs.

For the past 7 years, he has been part of a team that is developing a mobile Planar Laser Induced Fluorescence (PLIF) and a mobile coherent anti-Stokes Raman spectroscopy system (CARS) for various wind tunnels and test facilities.

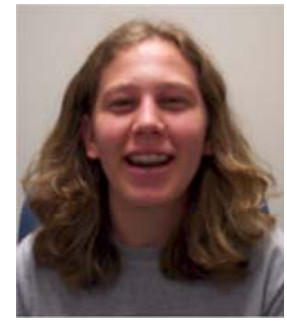

Sarah Tedder received her bachelor's degree in Aeronautical and Aerospace Engineering from Purdue University in 2003. While earning her degree at Purdue University she was a part of the NASA Co-op program beginning her work in 2000 at Kennedy Space Center and transferring the NASA Langley Research Center in the summer of 2001.

In 2004 she began her graduate studies in Physics at The College of William and Mary while working on coherent anti-Stokes Raman spectroscopy as graduate Co-op at NASA Langley. After receiving her Masters in 2006 she is currently continuing her studies towards a PhD. 


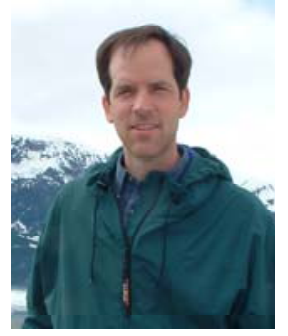

Paul Danehy received a BS in Mechanical Engineering from the University of New Hampshire in 1989 and $\mathrm{MS}$ and $\mathrm{PhD}$ degrees in Mechanical Engineering from Stanford University in 1991 and 1995 respectively. Part of his Ph.D. thesis work was completed at the Combustion Research Facility at Sandia National Laboratories. Between 1995 and 2000 he worked in the Physics Department at the Australian National University in Canberra, Australia, first as a post-doc and then as a faculty member. In 2000, he moved to the NASA Langley Research Center in Hampton, Virginia.

He currently works in the Advanced Sensing and Optical Measurement Branch where he leads research teams studying hypersonic flows using planar laser-induced fluorescence (PLIF) and developing coherent anti-Stokes Raman scattering (CARS) and Rayleigh scattering to study supersonic combustion.

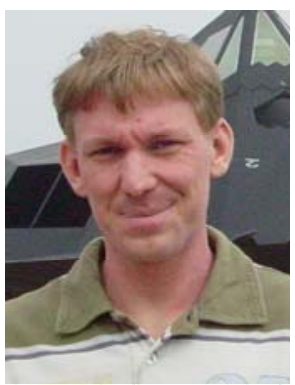

Markus C. Weikl studied Chemical Engineering at the Friedrich-Alexander Universität ErlangenNürnberg in Erlangen, Germany. After receiving his diploma degree, which is equivalent to a MS, in 2004 he continued his studies towards a PhD at the Department of Engineering Thermodynamics (LTT) in Erlangen.

His research interests are pure rotational and vibrational coherent anti-Stokes Raman scattering (CARS) and linear Raman scattering spectroscopy and their application to combustion systems. In 2006 he was invited as a guest researcher to the NASA Langley Research Center in Hampton, Virginia, where he helped on developing the combined interferometric Rayleigh scattering - coherent anti-Stokes Raman spectroscopy (CARS) system.

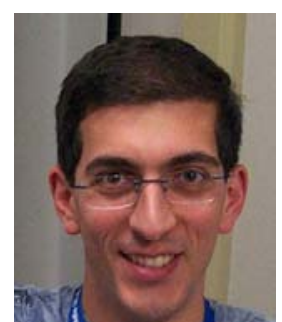

Gaetano Magnotti obtained his Bachelor Degree in Aerospace Engineering from the University of Naples “Federico II”, Italy, in 2004.

Currently is pursuing a doctoral degree in aerospace engineering at The George Washington University and conducting research at NASA Langley. His interests are scramjet propulsion, ground test facilities development and laser based measurements techniques.

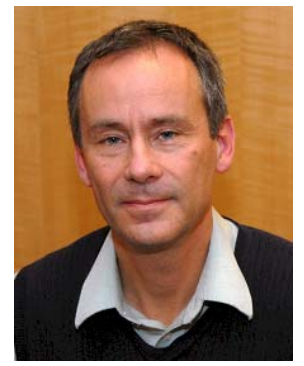

Andrew D. Cutler Andrew D. Cutler is a Professor in the Department of Mechanical and Aerospace Engineering at the George Washington University. He received his BSc degree in Mechanical Engineering at Imperial College, University of London in 1979, and his MS and PhD degrees in Mechanical Engineering at Stanford University in 1980 and 1984 respectively. He conducted postdoctoral research at Imperial College in the Department of Aeronautics before joining the George Washington University.

He conducts research in supersonic combustion, combustion diagnostics, and in various areas related to scramjet propulsion in the laboratories of the Hypersonic Air breathing Propulsion Branch at NASA Langley Research Center. 